JOPAT 14,$2009 ; 49$ - 54

\title{
The Benefit of HIV Voluntary Counseling and Testing (HVCT) in Abuja, Nigeria
}

\author{
Ya'aba $\mathrm{Y}^{1}$, Isu $\mathrm{NR}^{3}$, Isah $\mathrm{YV}^{2}$, Oladepo $\mathrm{DK}^{1}$, Mohammed $\mathrm{SB}^{1}$, \\ Ibrahim $\mathrm{K}^{1}$, Izebe $\mathrm{KS}^{1}$, Oladosu $\mathrm{P}^{1}$ and Onoja $\mathrm{AJ}^{4}$ \\ ${ }^{1}$ Department of Microbiology, Human Virology and Biotechnology, \\ National Institute for Pharmaceutical Research and Development (NIPRD) Idu-Abuja, Nigeria \\ ${ }^{2}$ FACA, Abuja, Nigeria. \\ ${ }^{3}$ Department of Biological Sciences, University of Abuja, Nigeria. \\ ${ }^{4}$ African Health Project, Abuja.
}

\begin{abstract}
HIV/AIDS present a major crisis that is increasingly affecting the most productive segments of the population across the development sectors in Nigeria. HIV voluntary counselling and testing (HVCT) has demonstrated its ability to increase number of people coming for testing because its free, the use of care and support services among adults, has contributed to viable strategies of HIV interventions. HIV antibody rapid testing has emerged as a key component of its prevention strategies world wide. Despite its importance in reducing HIV risk behavior and linking people to care and treatment, the actual HIV antibodies testing is still relatively low. Some rural areas in three out of six area councils in the Federal Capital Territory, Abuja were visited from November 2006 to January 2007. One thousand six hundred and seventy (1670) people were voluntarily counselled and tested in these areas. Serial algorithm was performed using Determine HIV1\&2 (Abbott Japan Co., LTD. Minato-ku,Tokyo, Japan.), Stat pak ${ }^{\mathrm{TM}}$ HIV1\&2 (Chembio diagnostic system, Inc. 3661 Horseblock road Medford, NY 11763, USA.) and Genie II HIV-1/HIV-2 (Bio-Rad, 3 boulevard Raymond Poincare 92430 Marnes LA Coquette- France). 734 (43.95\%) were male and 936 (56.05\%) were female. $115(6.89 \%)$ people were positive for HIV antibodies, $1392(83.35 \%)$ were negative for HIV antibodies and $163(9.76 \%)$ defaulted. $1587(95 \%)$ had never tested their HIV status benefitted from these programme. It is necessary to continue HVCT awareness in rural areas to avail more opportunity to benefit from these services to reduce HIV/AIDS infection.
\end{abstract}

Keywords: HVCT, HIV, Determine, Stat pak ${ }^{\mathrm{TM}}$, Genie II, Rural areas

\begin{abstract}
Introduction
HIV/AIDS infection continues to pose a major threat to many people in Nigeria. It is now apparent that HIV/AIDS epidemic is a national disaster and therefore, everyone's problem and that is the reason why our government responded to the expressed and unexpressed needs of the research institute, non governmental organizations (NGOs), university community and the needs of its broader external community. Various activities undertaken have demonstrated the need for a concerted intervention requiring creative, well-informed leadership from a section potentially wellresourced to shape institutional and inter-institutional responses.
\end{abstract}

Since 1985, when a test for HIV antibodies first became available [5, 9], issues pertaining to HIV testing behaviour have been of considerable interest to research. Some of the issues raised in previous studies are the benefits of testing for HIV antibodies. One of the benefits is the reduction of risky sexual behaviour $[8,10]$ and allowing early access to clinical care $[3,4]$.

* Corresponding Author: Email: yakyabnig@yahoo.com 
HIV voluntary counselling and testing (HVCT) is the process by which a person undergoes counselling enabling him or her to cope with stress and make informed choices about HIV testing. HVCT programs have demonstrated their ability to increase safe sexual behavior and use of care and support services among adults [2]. By helping clients learn their HIV sero status and creating a personalized HIV risk reduction plan, HVCT can provide the information and support necessary to change risky behaviors that could lead to HIV infection or transmission [1].Pre counselling of subjects, post counselling of subjects and a risk reduction plan are the key features that distinguish HVCT from other HIV testing services.

HVCT has become a widely advocated HIV/AIDS prevention strategy among adults. Most clients of HVCT have become advocates for HIV/AIDS prevention strategy. Sixty percent of all new HIV infections in sub-Saharan Africa, however, occur among young people between the ages of 10 to 24 [11].

The focus of this study is to ascertain the relevance and benefit of HIV voluntary counselling and testing (HVCT) in rural areas of Abuja, Nigeria. We chose rural areas because of the profound effects of HIV/AIDS and lack of awareness in this setting.

\section{Methods}

This study was cross-sectionally designed and conducted in the Federal Capital Territory (FCT) Abuja. Abuja is the developing Federal Capital City of Nigeria. It is located at the centre of the country with a land area of 8,000 square meters. It has boundary with the Kaduna state on the North, Plateau State in the East and South East, Kogi State to South West and Niger State to the West. It is divided into six area councils; Abaji, Gwagwalada, Kuje, Municipal, Bwari and Kwali. It was a purposely built capital city to replace Lagos, and it officially replaced Lagos in December 1991, after 15 years of planning. This research work was funded by FCT Action Committee on AIDS (FACA), Abuja, from November, 2006 to January, 2007 in three out of a total of six Area councils. The research instrument, originally constructed in English, was translated into Hausa (Language spoken widely in Abuja, FCT) and then back- translated into English to ensure validity of questions. The participants gave their consent prior to the study; however consent was gotten on behalf of those below 18years from their parents or guardians.

One thousand six hundred and seventy (1670) people were voluntarily counselled and tested in these rural areas. The HIV rapid testing was performed in line with the national algorithm using Determine HIV1\&2 (Abbott Japan Co.,), Stat pak ${ }^{\mathrm{TM}}$ HIV $1 \& 2$ (Chembio diagnostic system, Inc) and Genie II HIV-1/HIV-2 (Bio-Rad, 3 Boulevard Raymond Poincare 92430 Marnes LA Coquette- France) onsite and test results were offered to the clients the same day. A client was considered to be HIV positive if he/she had two positive rapid HIV test or positive tie breaker (Genie 11 HIV1/HIV2) in cases where rapid test results were ambiguous or discordant. 


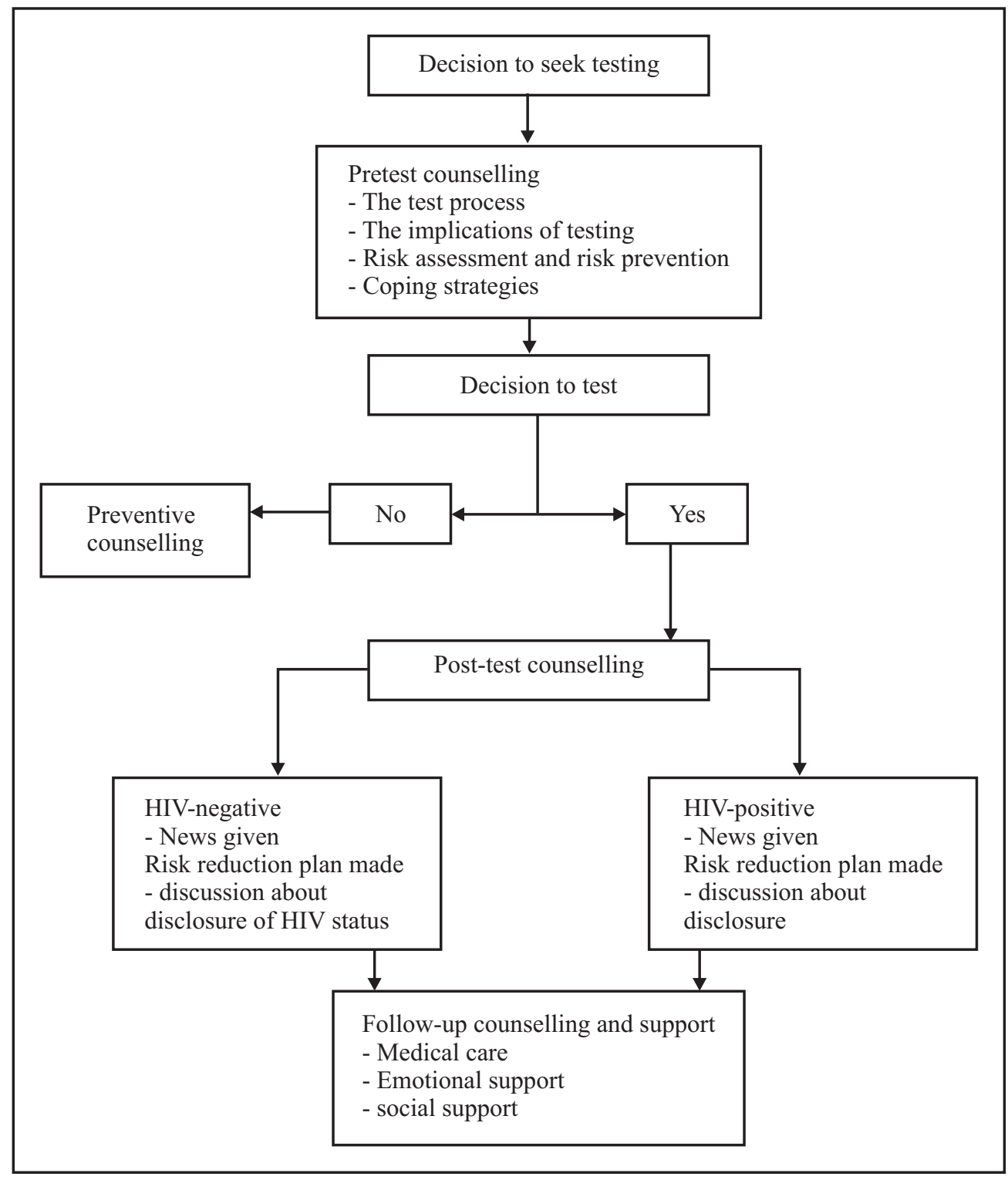

Figure 1: HVCT MODEL [6]

\section{Results}

One thousand six hundred and seventy $(1,670)$ subjects were counseled and tested for HIV antibodies. $734(43.95 \%)$ were male and $936(56.05 \%)$ were female with mean age of 34.115 (6.89\%) were positive for HIV antibodies, $1392(83.35 \%)$ were negative. $163(9.76 \%)$ declined testing despite the counselling. One thousand five hundred and eighty-seven $(1,587)$ had never known their HIV status before (Table 1). The results of One thousand Five hundred and Seven(1507) clients screened for HIV shows that Seven (7.6\%) were positive for HIV. Twenty nine (1.9\%) were males and Eighty six (5.7\%) were females. It is seen that highest prevalence of HIV was obtained amongst the age bracket (26-30) and followed by age bracket (31-35) as shown in figure 2 . 
Table 1: Sociodemographic characteristics of studied population

\begin{tabular}{llll}
\hline Sociodemographic & No. of Clients & Percentage (\%) \\
\hline Sex & Male & 734 & 44 \\
& Female & 936 & 56 \\
\hline Currently in school & & \\
& Yes & 1106 & 66 \\
& No & 564 & 34 \\
\hline Marital status & & & 51 \\
& Single & 856 & 44 \\
& Married & 726 & 5 \\
\hline Divorced & 88 & 85 \\
& & & 15 \\
\hline Ever had sex & Yes & 1414 & \\
& No & 256 & 5 \\
& & 95 \\
\hline HIV testing previous & 83 & 90 \\
& Yes & 1587 & 10 \\
\hline HVCT & No & 1507 &
\end{tabular}

Note: Percentage may not add up to $100 \%$ since percentage were rounded to nearest whole.

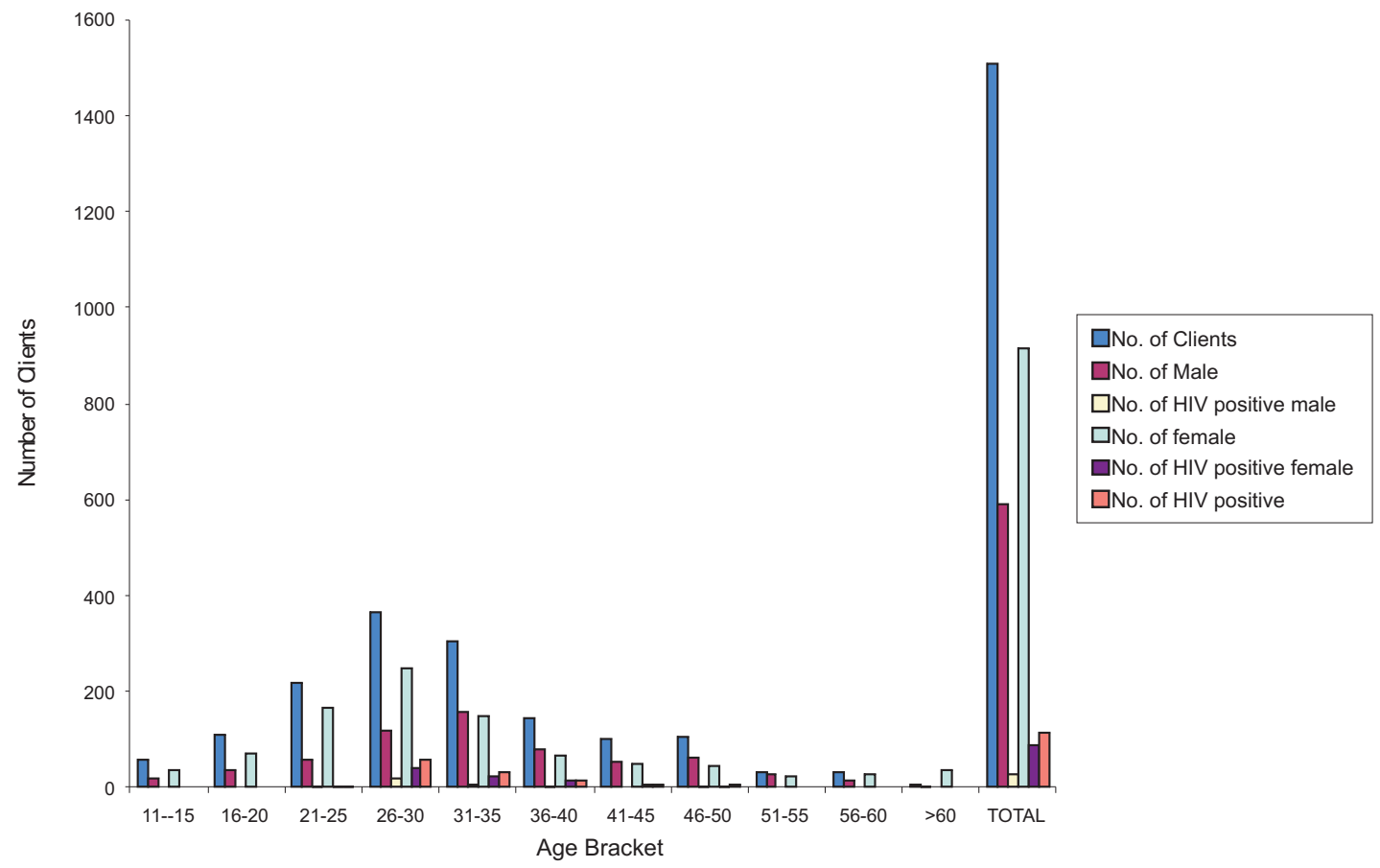

Figure 2: The Prevalence of HIV infection in Abuja, Nigeria. 


\section{Discussion and Conclusion}

The results obtained were quite interesting. A staggering $95 \%(1,587)$ of the selected population did not know their HIV status. Of this population studied $163((9.76 \%)$ of the population studied declined testing despite counselling (Table 1). Reasons given ranged from ignorance of the benefits of knowing their HIV status, fear of alienation and stigmatization. 3.52\% refused testing at first but returned a few days after counselling for testing. This further stresses the need to continue awareness programmes for individuals in rural areas to know their HIV serostatus. This benefit would create an HIV risk reduction plan, since individuals migrate from rural to urban and vise versa for survival.

Sexually active age group is a factor that predisposes people to HIV infection and high rate of infection is expected in such groups. Age group with the highest infection was among both males and female of age bracket 26-30 and 31-35 years with 18, 7 persons infected with HIV respectively while that of females was at age bracket of 26-30 and 31-35 years had 39,23 cases. Data showed an increase in infection with age and peaked at the age bracket 26-30 after which a decrease followed and no infection was observed in people higher than 51 years.

Barriers to accessing HVCT services include stigmatization, geographic accessibility, lack of social promotion, inefficient counselling and testing practices, and cost [12]. These studies have demonstrated that a period of HVCT significantly increased the number of persons testing per day and enhances cost-effectiveness of HVCT when offered as an integrated program within an existing AIDS service-oriented non governmental organization. While HIV testing behaviour and associated characteristics has been studied in the general population, sector specific information is largely lacking. As articulated by the International Labour Organization, studies that contribute specifically to the multilateral effort to combat HIV/AIDS at workplaces are needed [7].

Therefore, integration of this HVCT program within a volunteer-based AIDS service organization should also be encouraged.

\section{Acknowledgment}

We thank the FCT Action Committee on AIDS (FACA) for funding this work and National Institute for Pharmaceutical Research and Development (NIPRD) for their support.

\section{References}

1. Centers for Disease Control and Prevention (CDC) (1994). HIV Counselling, Testing and Referral Standards and Guidelines. Atlanta, GA: U.S. Department of Health and Human Services.

2. Coates, T. (1998). "Voluntary HIV counselling and testing (VCT) reduces risk behavior in developing countries: Results from the voluntary counselling and testing study," Paper presented at the International Conference on AIDS, Geneva, Switzerland, 28 June -3 July.

3. Day, J.H., Miyamura, K., Grant, A.D., Leeuw, A., Munsamy, J., Baggaley, R. and Churchyard, G.J (2003). Attitudes to HIV voluntary counselling and testing among mineworkers in South Africa: will availability of antiretroviral therapy encourage testing $\square$ AIDS Care, 15: 665-672.

4. Gayle, H. and Lange, J.M. (2004). Seizing the opportunity to capitalise on the growing access to HIV treatment to expand HIV prevention. Lancet, 364: 6-7

5. Holtzman, D., Rubinson, R., Bland, S. and Mcqueen, D. (1998). HIV testing behaviour and associated characteristics among U.S. adults, 1993 and 1994. AIDS and Behavior, 2: 269-281.

6. Horizon Program (2001). HIV Voluntary Counselling and Testing among youth. Result from an exploratory study in Kenya (Nairobi) and Uganda (Kampala and Masaka) Pp.10.

7. ILO (2004). HIV/AIDS and work: global estimates, impact and response (Revised Edition). The ILO program on HIV/AIDS and the world of work. Switzerland: ILO.

8. Painter, T.M. (2001). Voluntary counselling and testing for couples: a highleverageintervention for HIV/AIDS prevention in sub-Saharan Africa. Social Science and Medicine, 53: 1397-1411. 
9. Phillips, K.A. and Coates, T.J. (1995). HIV counselling and testing: research and policy issues. AIDS Care, 7: 115-124.

10. Sangiwa, M.G., Van Der Straten, A. and Grinstead, O. (2000). Client's perspective of the role of voluntary counselling and testing in HIV/AIDS prevention and care in Dar Es Salaam, Tanzania. AIDS and Behavior, 4: 35-48.

11. UNAIDS (1998). "Force for change: World AIDS campaign with young people." 1998 World AIDS Campaign Briefing Paper. http://www.unaids.org/unaids/events/wad/1998/force\%2de.doc.

12. Vermund SH and Wilson CM (2002). Barriers to HIV testing-where next $\square$ Lancet. 360:1186-1187. 